\title{
Magnetic network dynamics: Activity level, feature size and anchoring depth
}

\begin{abstract}
N. Meunier
Laboratoire d'Astrophysique de l'Observatoire Midi-Pyrénées, 57 avenue d'Azereix, BP 826, 65008 Tarbes Cedex, France

e-mail: meunier@bagn.obs-mip.fr

Received 23 November 2004 / Accepted 16 February 2005

Abstract. A large data set of MDI magnetograms is studied in order to analyze the global dynamics of magnetic structures from network to active regions. Two complementary techniques are used: feature tracking and correlation tracking. The very large data set allows the variation of their dynamics (angular rotation velocity and meridional circulation) to be studied in detail. Variation both in the dynamics as a function of size and distance to active regions (for feature tracking) and as a function of activity level in the correlation box are studied. These new results allow the possible anchoring depth of these magnetic features to be constrained. The observations of the magnetic network dynamics are not consistent with the dynamics deduced from helioseismology. So, some additional processes must be acting on the magnetic network in order to explain their global dynamics.
\end{abstract}

Key words. Sun: magnetic fields - Sun: photosphere - Sun: faculae, plages

\section{Introduction}

The differential rotation of the most active solar features (spots and plages) have been studied extensively over the past decades. The various methods and data selection have led to angular rotation velocity (hereafter rotation rates) of various amplitudes (see for example Howard 1996; Beck 2000, for reviews). These differences have long been interpreted in terms of anchoring depth of these features, since structures anchored in layers rotating differently should rotate at different rates as well. Before the advent of helioseismology this interpretation was used to obtain some clues about the internal rotation rate. The opposite approach can now be used, such that it is possible to try to determine the anchoring depth of magnetic features from their rotation rate. Javaraiah \& Gokhale (1997), Hiremath (2002), and Sivaraman \& Gokhale (2004) have thus used the internal rotation inferred by helioseismology to determine the anchoring depth of spots during their lifetimes. However, this interpretation of the different rotation rates of magnetic structures by their anchoring in the convection zone has never been completely analyzed, especially using large data sets and several dynamical variables. One should also remember that the faster rotation rate of sunspots, compared to the photospheric plasma, has also been explained by D'Silva \& Howard (1994) as the interaction between magnetic buoyancy and drag and the Coriolis force acting on the rising flux tubes. Therefore there is a need for analysis of global dynamics data in order to check the consistency of the interpretation.

In this paper, the angular rotation velocity (in heliographic degrees per day) of magnetic structures from network to large regions is studied using two complementary methods, where measurement of the meridional circulation is used as an additional constraint. We focus mainly on magnetic network analysis since fewer work has been done for these less active structures. The works of Snodgrass \& Ulrich (1990), Komm et al. (1993a,b), Meunier et al. (1997a,b), and Meunier (1999) did not study the variation of the dynamics of the magnetic network in detail and did not address the issue of anchoring depth. Variation in the rotation rate and meridional circulation provides additional constraints. In Sect. 2, the methods and limitations are described. Section 3 shows the time-averaged results, with the dependence of the dynamics on latitude, on the type of features, and on their location with respect to active regions. Finally a discussion in terms of anchoring depth is presented in Sect. 4.

\section{Data and processing}

\subsection{MDI data}

A data set of full-disk magnetograms obtained by MDI on SOHO (Scherrer et al. 1995) between June 22, 1996 and April 1, 2004 is used, covers 8 years, i.e. most of cycle 23 from cycle minimum to the end of cycle maximum. For this study, I have selected only 5-min averaged magnetograms as in Meunier (2003), in order to have a homogeneous data set. These magnetograms, all at level 1.8, are separated by $96 \mathrm{~min}$ or a multiple of $96 \mathrm{~min}$. The distribution of image pairs with time is shown in Fig. 1 and is exactly the same for both methods described below. There are a few gaps because during some 


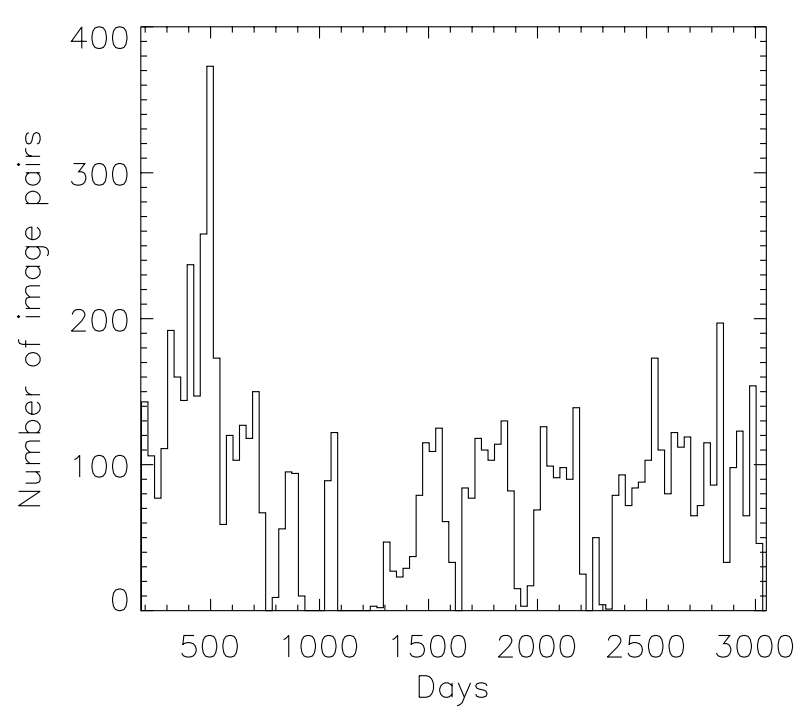

Fig. 1. Distribution of image pairs versus time. Day 1 corresponds to January 1, 1996.

periods only 1-min magnetograms are available. One important gap is also due to the interruption of SOHO activities in 1998. For a separation of $96 \mathrm{~min}, 8117$ image pairs are thus available.

\subsection{Feature tracking}

Feature identification is performed as in Meunier (2003), using a $40 \mathrm{G}$ threshold on $B / \mu$ maps, where $B$ is the measured lineof-sight magnetic field (i.e. a magnetic flux averaged over the pixel size) and $\mu$ the cosinus of the angle between the line-ofsight direction and the normal to the solar surface. Note that this threshold of $40 \mathrm{G}$ is quite conservative and is well above noise level in these data (below $10 \mathrm{G}$ ). Following this identification on each image, an association between features observed on two consecutive images separated by $96 \mathrm{~min}$ is performed. For each feature on the first image, the closest feature is identified once shifted by a reference synodic rotation rate of $13.5-3.5 \sin ^{2} \theta \mathrm{deg} /$ day ( $\theta$ latitude). Only feature pairs are selected such that this distance $d$ is below $4.5 \mathrm{Mm}$ (which corresponds to a diffusion coefficient of $1000 \mathrm{~km}^{2} \mathrm{~s}^{-1}$ ) and such that the size is not modified by more than $5 \%$. The threshold of $4.5 \mathrm{Mm}$ derives from the distribution of $d$, which shows a peak around $1.2 \mathrm{Mm}$, then a minimum (much smaller than the maximum) around 4.5 Mm and increases slightly again. The second distribution above $4.5 \mathrm{Mm}$, visible mostly for small structures, corresponds to cases where the structure has either disappeared or split on the second magnetograms and is therefore identified with a random structure anywhere on the disk. The 2 distributions are distinct, and therefore it is quite safe to consider that below 4.5 Mm the tracking is correct. The distribution of $d$ up to $4.5 \mathrm{Mm}$ is shown in Fig. 2. The maximum of $1.2 \mathrm{Mm}$ would correspond to a diffusion coefficient of $250 \mathrm{~km}^{2} \mathrm{~s}^{-1}$. One should note that for the smallest structures $\left(10 \mathrm{Mm}^{2}\right), 4.5 \mathrm{Mm}$ corresponds to 2.5 times the radius of this structure and only 3 pixels. A distance of $4.5 \mathrm{Mm}$ corresponds to the radius of structures with a size of $63 \mathrm{Mm}^{2}$. We have checked that the results are not affected significantly

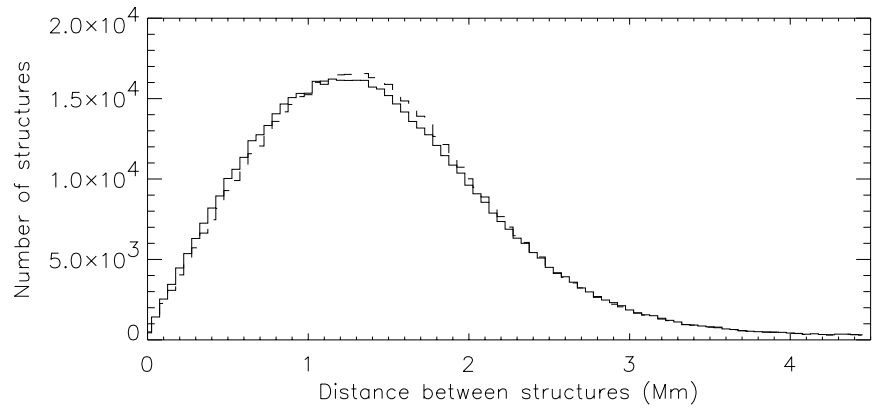

Fig. 2. Distribution of distances between two associated magnetic features in the FT method for sizes below $80 \mathrm{Mm}^{2}$ (solid line) and all structures (dashed line).

by this selection. In addition, the use of a different reference rotation rate (for example an equatorial rate of 13.3 or $13.7 \mathrm{deg} /$ day instead of $13.5 \mathrm{deg} /$ day, or a coefficient very different from $-3.5 \mathrm{deg} / \mathrm{day}$ ) does not change the results, i.e. the tracking appears to be very robust.

This feature tracking method is hereafter denoted as FT. In order to look at the dependence of the dynamics on the type of structure, additional variables are computed:

- The size $A_{\mathrm{t}}$ of the structure, defined by the $40 \mathrm{G}$ threshold on $B / \mu$, in $\mathrm{Mm}^{2}$.

- The perimeter $P_{\mathrm{t}}$ of the structure, defined by the $40 \mathrm{G}$ threshold on $B / \mu$, in $\mathrm{Mm}$.

- The flux in that structure, $\Phi_{t}$, in Mx (unsigned).

- The maximum absolute magnetic field in that structure, $B m_{\mathrm{t}}$, in $\mathrm{G}$.

\subsection{Correlation tracking}

The correlation tracking (hereafter denoted as CT) is performed as in Meunier (1999). I recall here the main characteristics: the cross-correlations are computed over boxes of $7.68 \times 7.68 \mathrm{deg}^{2}$ $\left(64 \times 64\right.$ pixels $\left.^{2}\right)$ on remapped magnetograms (1 pixel corresponds to 0.12 degrees, i.e. $1.45 \mathrm{Mm}$ at disk center). Individual cross-correlation functions are then fitted with 2D Gaussians, which provides the rotation rate and the meridional circulation. Only boxes with maximum correlation above 0.2 are considered in this analysis.

In order to look at the dependence of the dynamics on the type of regions, additional variables are computed:

- The average of the absolute magnetic field in the box $\left\langle\left|B_{\mathrm{c}}\right|\right\rangle$ in $\mathrm{G}$.

- The maximum absolute magnetic field in that structure $B m_{\mathrm{c}}$ in $\mathrm{G}$.

- The number of pixels above the $40 \mathrm{G}$ threshold in the box $N$ pix .

\subsection{Additional computation}

Corrections from synodic rotation rates to sidereal rotation rates are performed. The residuals of the rotation rate and meridional circulation are computed with respect to the average value in the corresponding latitude range $\left(5^{\circ}\right.$ bin) averaged 
over the full time period and all points. The sign convention is as follows for the meridional circulation: a positive circulation means motion toward the north, i.e. it is poleward in the Northern hemisphere and equatorward in the Southern hemisphere. The convention is changed for the residual circulation to make it easier to average values over both hemispheres: a positive residual is positive when it is more poleward than the average, and negative otherwise. Note that for the equator, the notion of poleward and equatorward motions does not make sense and only the notion of southward or northward motions is usefull.

\subsection{Possible biases}

\subsubsection{Variation of the rotation rate with longitude}

In Meunier (1999), a bias in longitude has been observed and studied. The rotation rate was observed to be larger on the west side than on the east side. We refer to that paper for the main characteristics. This bias is shown in Fig. 3 for various feature sizes (FT method) and various magnetic field levels (CT method). These plots show that the strong variation in rotation with longitude is mostly visible for CT and only for quiet regions. It has been suggested (Giles 1999) that variation of the plate scale across the disk in MDI data could lead to such a result. However, if this were true, the effect would probably be visible for all features and both methods.

That this is seen only for weak field regions suggests that the noise level, which is also strongly varying on the solar disk in MDI data, could play a part in the problem. However, as the noise is larger far from disk center, one expects a small rotation residual on the east and west sides (with a stronger effect on the west side where the noise gradient is larger) and a larger rotation residual at disk center, which is not what is observed. Also, Meunier (1999) observed that the amplitude of the bias was larger for 5-min magnetograms compared to single ones, although the former are less noisy.

As a consequence, the origin of this bias remains unclear. It does not correspond to any of the possible biases studied extensively by Strous (2000), and is almost certainly of instrumental origin though. Therefore the CT results presented in this paper have been corrected for this bias as observed in Fig. 3: the bias for the rotation rate is computed for different ranges in magnetic field for the whole period and then subtracted from the original rotation rate. However the results are not significantly affected by this correction. Furthermore, we checked that a subset of structures located close to disk center led to similar results.

\subsubsection{Non-zero disk-center meridional circulation}

A non-zero meridional circulation at the equator has already been observed with MDI data using various techniques (Meunier 1999; Giles 2000, for example). Here, for the FT method, the average meridional circulation at the equator is $-0.051 \pm 0.003 \mathrm{deg} / \mathrm{day}(-0.047 \pm 0.002 \mathrm{deg} /$ day for $\mu>0.95)$. For the CT method, the average meridional circulation at the equator is $-0.034 \pm 0.002 \mathrm{deg} / \mathrm{day}(-0.012 \pm 0.001 \mathrm{deg} / \mathrm{day}$
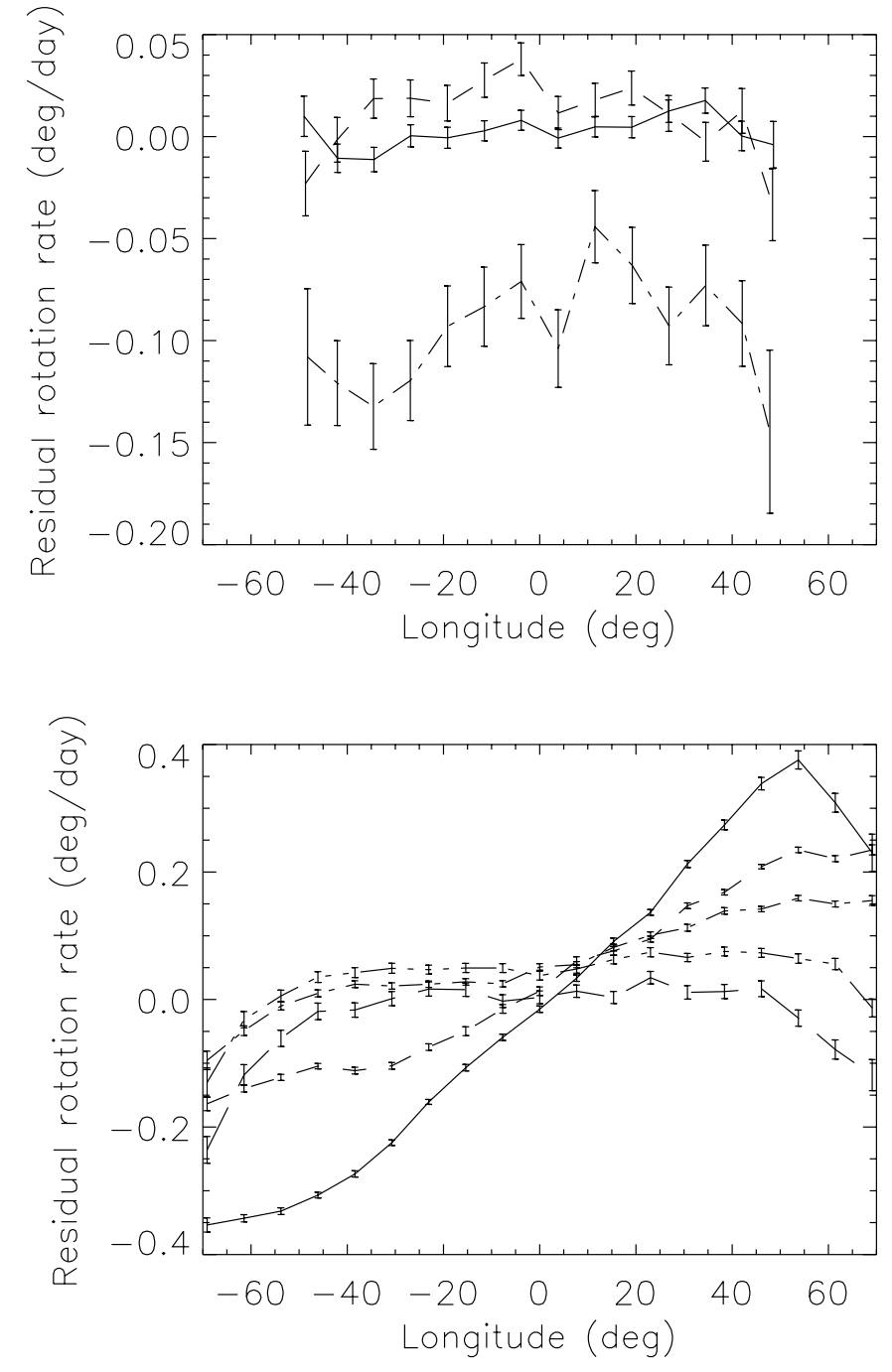

Fig. 3. Top: FT residual rotation rates versus longitude for $A_{\mathrm{t}}<$ $80 \mathrm{Mm}^{2}$ (solid), $A_{\mathrm{t}}$ in the range $80-500 \mathrm{Mm}^{2}$ (dashed), and $A_{\mathrm{t}}>$ $500 \mathrm{Mm}^{2}$ (dotted-dashed). Bottom: CT residual rotation rate versus longitude for $\left\langle\left|B_{\mathrm{c}}\right|\right\rangle$ below $6 \mathrm{G}$ (solid), in the range 6-9 $\mathrm{G}$ (dashed), in the range 9-15 G (dotted-dashed), in the range 15-30 G (dot-dot-dotdashed), and above $30 \mathrm{G}$ (long dashed).

for $\mu>0.95$ ). In addition to an average offset of a few $\mathrm{m} / \mathrm{s}$ (toward the South Pole), this offset is observed to vary with a period of approximately 1 year, suggesting influence from the SOHO orbit. It has been suggested that this could in part be due to a misalignment of the MDI CCD. Giles (1999) has also suggested that the $\mathrm{B}$ and $\mathrm{P}$ angles should be corrected in order to suppress this equatorial flow; but so far they are uncertain, and there is no way at this time to be sure that this apparent flow across the equator is not real. The different amplitudes for the 2 methods show that it cannot be due to an instrumental problem alone; otherwise they would be similar. Therefore the results presented in the following sections are not corrected for it. This uncertainty makes the comparison with helioseismic results a little more difficult because it introduces a north-south asymmetry that may or may not be real. 

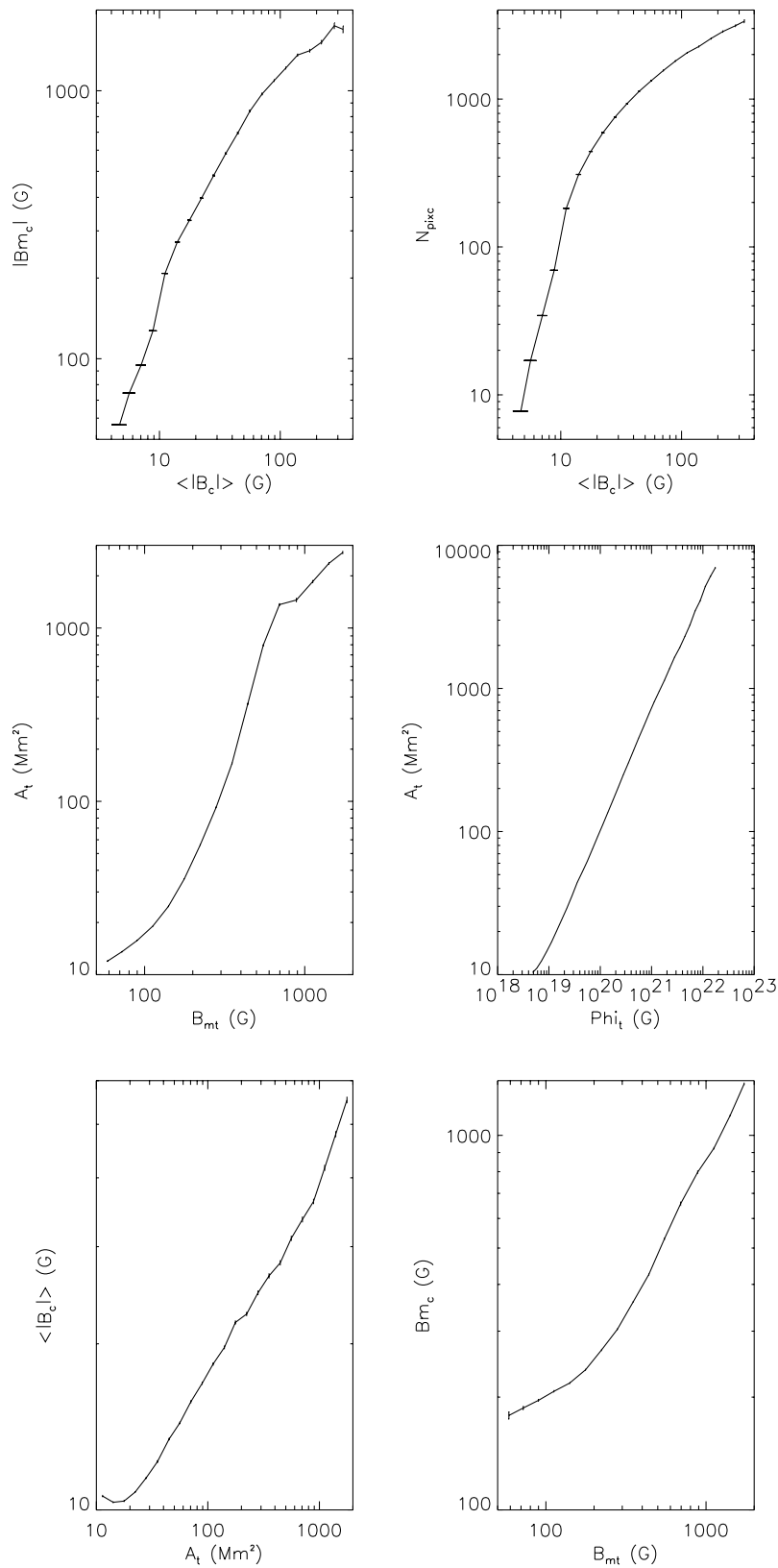

Fig. 4. Top: CT correspondences, showing $B m_{\mathrm{c}}$ versus $\left\langle\left|B_{\mathrm{c}}\right|\right\rangle$ (left) and $N$ pix $_{\mathrm{c}}$ versus $\left\langle\left|B_{\mathrm{c}}\right|\right\rangle$ (right). Middle: FT correspondences showing $A_{\mathrm{t}}$ versus $B m_{\mathrm{t}}$ (left) and $A_{\mathrm{t}}$ versus $\Phi_{\mathrm{t}}$. Bottom: FT-CT correspondences showing $\left\langle\left|B_{\mathrm{c}}\right|\right\rangle$ versus $A_{\mathrm{t}}$ (left) and $B m_{\mathrm{c}}$ versus $B m_{\mathrm{t}}($ right $)$.

\subsection{Correspondence between the two methods}

In order to help in interpreting the results, it is useful to build a correspondence between the characteristics of a given box used for correlation tracking, such as the average magnetic field or the number of pixels above $40 \mathrm{G}$ and the properties of individual features in that box, such as the average size of the features in that box or their average magnetic field. Note that in some cases, the correlation tracking method provides a result in very weak field regions where no feature above the magnetic threshold of $40 \mathrm{G}$ has been tracked. For the other cases, the results are shown in Fig. 4. Magnetic fields and sizes are naturally well correlated (Meunier 2003).
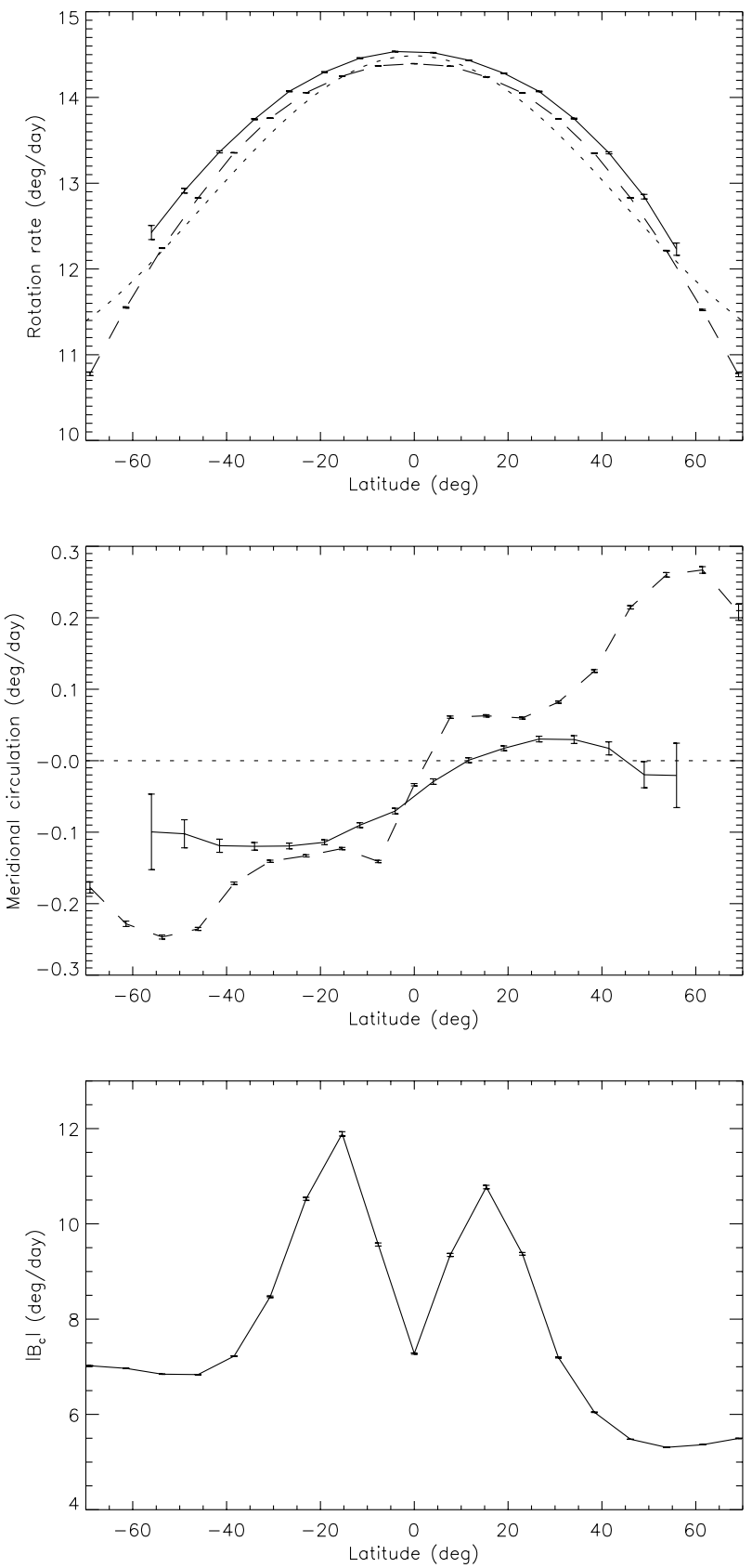

Fig. 5. Top: sidereal rotation rate for FT (solid line) and CT (dashed line); the dotted line represents the reference rotation rate around which the correct value is searched. Middle: same for the meridional circulation. Bottom: averaged $\left\langle\left|B_{\mathrm{c}}\right|\right\rangle$ showing active latitude regions. $0.1 \mathrm{deg} /$ day corresponds to $14 \mathrm{~m} / \mathrm{s}$.

\section{Time-averaged results}

\subsection{Rotation rate and meridional circulation versus latitude}

Figure 5 shows the rotation rates obtained for all regions for the 2 methods. Note that both are very different from the "reference" rotation rate. The CT provides a rotation rate which is smaller than the FT rotation rate at all latitudes by about $0.17 \mathrm{deg} / \mathrm{day}$. In the first case the results are compatible with 
Table 1. Equatorial rotation rate (in deg/day) from different works: photospheric magnetic features using CT or FT, except for: the work of Brajša et al. (2004), which concerns coronal bright points (Method 1 is interactive and selects less features in latitude range $10-40^{\circ}$ than the automatic Method 2); The work of Meunier et al. (1997) is from CaII photospheric images. Supergranulation (denoted as SG) is from autocorrelation of dopplergrams. Coefficients $A_{2}$ and $A_{4}$ are Legendre coefficients from the fit on the rotation versus latitude.

\begin{tabular}{ccccc}
\hline \hline Reference & Period & Rotation rate & $B$ & $C$ \\
\hline CT present work & $1996-2004$ & $14.390 \pm 0.001$ & $-2.568 \pm 0.003$ & $-0.552 \pm 0.003$ \\
CT Meunier (1999) & $1996-1998$ & $14.33 \pm 0.02$ & $-2.671 \pm 0.005$ & $-0.630 \pm 0.005$ \\
CT Komm et al. (1993a) & $1975-1991$ & $14.43 \pm 0.02$ & $-2.53 \pm 0.12$ & $-0.48 \pm 0.03$ \\
CT Snodgrass \& Ulrich (1990) & $1967-1987$ & $14.25 \pm 0.01$ & $-2.49 \pm 0.07$ & $-0.55 \pm 0.02$ \\
SG Duvall (1980) & 1979 & $14.72 \pm 0.07$ & - & - \\
SG Snodgrass \& Ulrich (1990) & $1967-1987$ & $14.71 \pm 0.05$ & $-2.62 \pm 0.19$ & $-0.41 \pm 0.06$ \\
FT present work & $1996-2004$ & $14.562 \pm 0.002$ & $-2.21 \pm 0.03$ & $-0.34 \pm 0.02$ \\
FT Meunier et al. (1997) & $1957-1964$ & $14.42 \pm 0.02$ & $-1.85 \pm 0.51$ & $0.16 \pm 0.20$ \\
Cor. BP Brajša et al. (2004) & $1998-1999$ & $14.454 \pm 0.027$ & $-2.65 \pm 0.36$ & $-0.32 \pm 0.11$ \\
Cor. BP Brajša et al. (2004) & $1998-1999$ & $14.677 \pm 0.033$ & $-2.52 \pm 0.42$ & $-0.63 \pm 0.13$ \\
\hline
\end{tabular}

previous results using a similar method (Snodgrass \& Ulrich 1990; Komm et al. 1993a; Meunier 1999), with an equatorial rotation rate of $14.39 \mathrm{deg} /$ day (see Table 1). In the FT case, no work for photospheric network features has been performed, except Meunier et al. (1997a) using a manual tracking of facula features observed on calcium images, including network magnetic fields: the equatorial rotation rate of $14.56 \mathrm{deg} / \mathrm{day}$ is larger than their results. However, the results are quite close to coronal bright point rotation rates (Brajša et al. 2004). Their automatic method provides a large equatorial rotation rate closer to FT than to CT results, and the interactive method is closer to the CT results. Note that the rotation rate obtained with their automatic method is also very close to that observed by Antonucci et al. (1979) for small-scale features in the corona.

Table 1 also shows a comparison of the Legendre coefficients $A_{2}$ and $A_{4}$, corresponding to the differentiality of the rotation (at low and high latitudes respectively). There is a tendency for the CT method to provide a more differential rotation than for the FT method. The large errorbars of the FT method in the literature prevents a detailed comparison, however. The CT coefficients seem significantly different, though quite close, but part of these differences could be due to a temporal variation. This is true in particular for the comparison of the present result with that of Meunier (1999), which shows a more differential rotation at cycle minimum.

The meridional circulation is also quite different for the 2 methods, as the FT provides a meridional circulation of much smaller amplitude $(7 \mathrm{~m} / \mathrm{s}$ instead of $35 \mathrm{~m} / \mathrm{s})$ than CT. The CT results are significantly larger than those obtained by Komm et al. (1993b) for example $(10-15 \mathrm{~m} / \mathrm{s})$, but these differences could be due to a time variation. The peculiar pattern observed with the CT in active latitudes (and already observed by Meunier 1999, who found that it corresponded to active regions) is not observed. The CT flow is observed to peak around latitudes $50-60^{\circ}$, while the FT peaks around $30^{\circ}$.
The pattern in the meridional circulation observed for $\mathrm{CT}$ is highly correlated with active latitudes. When looking at similar plots for various periods through the cycle, the location of this pattern is moving toward the equator similarly to the activity belt. Therefore the complex pattern is not due to superimposition of various components resulting from different periods. However, no clear pattern is observed for the FT analysis. Following the work of Howard (1991), we recomputed the FT meridional circulation with respect to the average meridional circulation found for the latitude that corresponds to the mean activity belt. For each time period of 30 days, the average latitude of structures larger than $200 \mathrm{Mm}^{2}$ in each hemisphere is computed, as well as the average meridional circulation within $3^{\circ}$ of that latitude. The meridional circulation in each hemisphere is then recomputed with respect to this average meridional circulation.

Results in both hemispheres are averaged together, with the following convention: a positive meridional circulation corresponds to a more poleward motion than the reference. Results are shown in Fig. 6. For small structures, a divergent pattern is observed with a stronger flow closer to the hemisphere than to the poles. The amplitude is quite low at a few $\mathrm{m} / \mathrm{s}$, as has been observed for other features such as recurrent sunspots using the same techniques (Wöhl \& Brajša 2001; Wöhl 2002). This signal is observed mainly for structures close to active regions (see Sect. 3.3.1) but becomes very small farther than $30^{\circ}$ from large structures. Note that we do not find the convergent pattern observed for faculae by Howard (1996). Large structures also exhibit a diverging pattern; however, poleward of the activity belt, the meridional circulation is constant within the errorbars.

The FT and CT methods are probably affected by the presence of active regions in different ways and in different proportions. Is this effect enough to explain the difference in results? To answer this question, the next section examines the variation in dynamics for various magnetic field regimes for both 


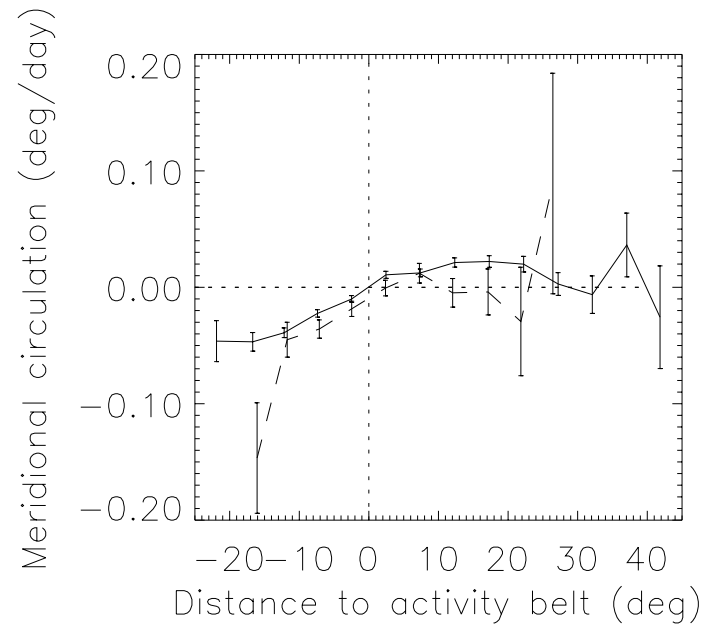

Fig. 6. FT meridional circulation with respect to the activity belt averaged over the two hemispheres. A positive signal means a more poleward motion than the reference. The solid line corresponds to all structures (dominated by small structures) and the dashed line to structures larger than $200 \mathrm{Mm}^{2}$.

methods independently, and then looks at the correspondence between the 2 methods using the results of Sect. 2.6.

\subsection{Dynamics versus structure size and magnetic field}

\subsubsection{Feature tracking}

Figure 7 shows the variation in residual rotation rates and residual meridional circulation with feature size $A_{\mathrm{t}}$, flux $\Phi_{\mathrm{t}}$, and maximum magnetic field in the structures $B m_{\mathrm{t}}$. We observe that the rotation rate of larger structures is smaller than for small features down to $40 \mathrm{Mm}^{2}$, i.e. network features. Such a trend has already been observed many times (see e.g. Howard 1990, 1992) for active regions, but this is the first time that this has been observed for network features down to $40 \mathrm{Mm}^{2}$. The slope is more pronounced for $A_{\mathrm{t}}$ above $\sim 700 \mathrm{Mm}^{2}\left(\Phi_{\mathrm{t}} \sim 8 \times 10^{20} \mathrm{Mx}\right.$ and $\left.B m_{\mathrm{t}} \sim 500 \mathrm{G}\right)$, and these 3 values correspond reasonably well (Fig. 4). Between 40 and $700 \mathrm{Mm}^{2}$, there is also a decrease in rotation rate with increasing size, but the slope is less pronounced; the curve even becomes a plateau for $\Phi_{\mathrm{t}}$ and $B m_{\mathrm{t}}$. For very small features below $40 \mathrm{Mm}^{2}$, as well as for low $\Phi_{\mathrm{t}}$ and low $B m_{\mathrm{t}}$, the rotation rate is again significantly smaller. Note that even when using a different reference rotation rate when performing the tracking, this result remains the same.

The meridional circulation residuals, on the other hand, are found to decrease from $10 \mathrm{Mm}^{2}$ to large active regions; i.e. large structures have a less poleward motion. Note that values around the equator were suppressed for this computation. The amplitude of the variation is much smaller than for the rotation rate.

It is important to keep in mind that a given size range may cover very different types of magnetic structures. For example, in Meunier (2003), it was found that in the range 2000-7000 $\mathrm{Mm}^{2}, 2$ populations were present with distinct distributions of $B m_{\mathrm{t}}$, some with $B m_{\mathrm{t}}$ above $900 \mathrm{G}$ corresponding to structures including spots while the others did not.
The population with large $B m_{\mathrm{t}}$ also had a much larger fractal dimension than the other one. Table 2 shows the dynamics for these two populations; the structures with spots rotate slower and exhibit less poleward motion than the other structures of similar size.

Structures of different sizes also represent a different evolutionary state. Emerging active regions, for example, are growing regions. Later they decay into smaller structures down to network features below $80 \mathrm{Mm}^{2}$. Therefore the largest structures are probably relatively old active regions, while very small structures like network features are older remnants. Structure of intermediary sizes probably cover a large range in age, however.

\subsubsection{Correlation tracking}

Figure 8 shows variation in the rotation rate and residual meridional circulation with average magnetic field $\left\langle\left|B_{\mathrm{c}}\right|\right\rangle$, with the number of pixels $N$ pix $_{\mathrm{c}}$ above $40 \mathrm{G}$ in the box, as well as with $B m_{\mathrm{c}}$. We observe a strong decrease of the residual rotation rate for $N$ pix ${ }_{\mathrm{c}}$ above 1000 pix, $\left\langle\left|B_{\mathrm{c}}\right|\right\rangle$ above $35 \mathrm{G}$ and $B m_{\mathrm{c}}$ above $700 \mathrm{G}$. These 3 levels correspond well to each other in Fig. 4. Starting from this level and going down to weaker magnetic field regions, we observe a decrease in the rotation rate residual, followed again by an increase up to $N$ pix ${ }_{\mathrm{c}} \sim 20$ pix, $\left\langle\left|B_{\mathrm{c}}\right|\right\rangle \sim 9 \mathrm{G}$ and $B m_{\mathrm{c}} \sim 80 \mathrm{G}$, and then again a decrease.

The meridional circulation residuals are decreasing for increasing $N$ pix $_{\mathrm{c}},\left\langle\left|B_{\mathrm{c}}\right|\right\rangle$, and $B m_{\mathrm{c}}$, which means that weak field areas have a more poleward motion. The decrease is monotonous for $N$ pix ${ }_{\mathrm{c}}$, but there is a tendency to increase again for $\left\langle\left|B_{\mathrm{c}}\right|\right\rangle$ above $100 \mathrm{G}$. A peak is also superimposed for $B m_{\mathrm{c}} \sim 400 \mathrm{G}$, due to the converging pattern observed in Fig. 5. Furthermore, when looking in detail at the meridional circulation, it is found that the decrease with increasing $\left\langle\left|B_{\mathrm{c}}\right|\right\rangle$ is observed everywhere except close to the equator (at latitudes $\pm 7.68^{\circ}$ ), where the gradient is reversed for $\left\langle\left|B_{\mathrm{c}}\right|\right\rangle$ lower than $10 \mathrm{G}$. This is consistent with the negative gradient observed by Komm et al. (2004) in a shallow layer (down to $1 \mathrm{Mm}$ ) close to the equator in the Northern hemisphere. However they did not observe it in the Southern hemisphere.

Figure 9 also shows the meridional circulation versus latitude for various magnetic field domains. It confirms the results of Meunier (1999), where the pattern of converging flows (with respect to the average) is observed only for strong field regions, i.e. active regions with $\left\langle\left|B_{\mathrm{c}}\right|\right\rangle$ in the range $10-50 \mathrm{G}$. Also, the variation with magnetic field is very strong at high latitude, with a much stronger poleward motion for weak field areas.

\subsubsection{Comparison of FT and CT}

For active regions, the most striking difference is for the meridional circulation, which exhibits very different patterns. However, one should keep in mind that with the CT, one box may see only a part of the active region, while the FT sees the whole structure. Therefore, the CT can probe more local flows in the case of very active regions compared to the CT; if there 

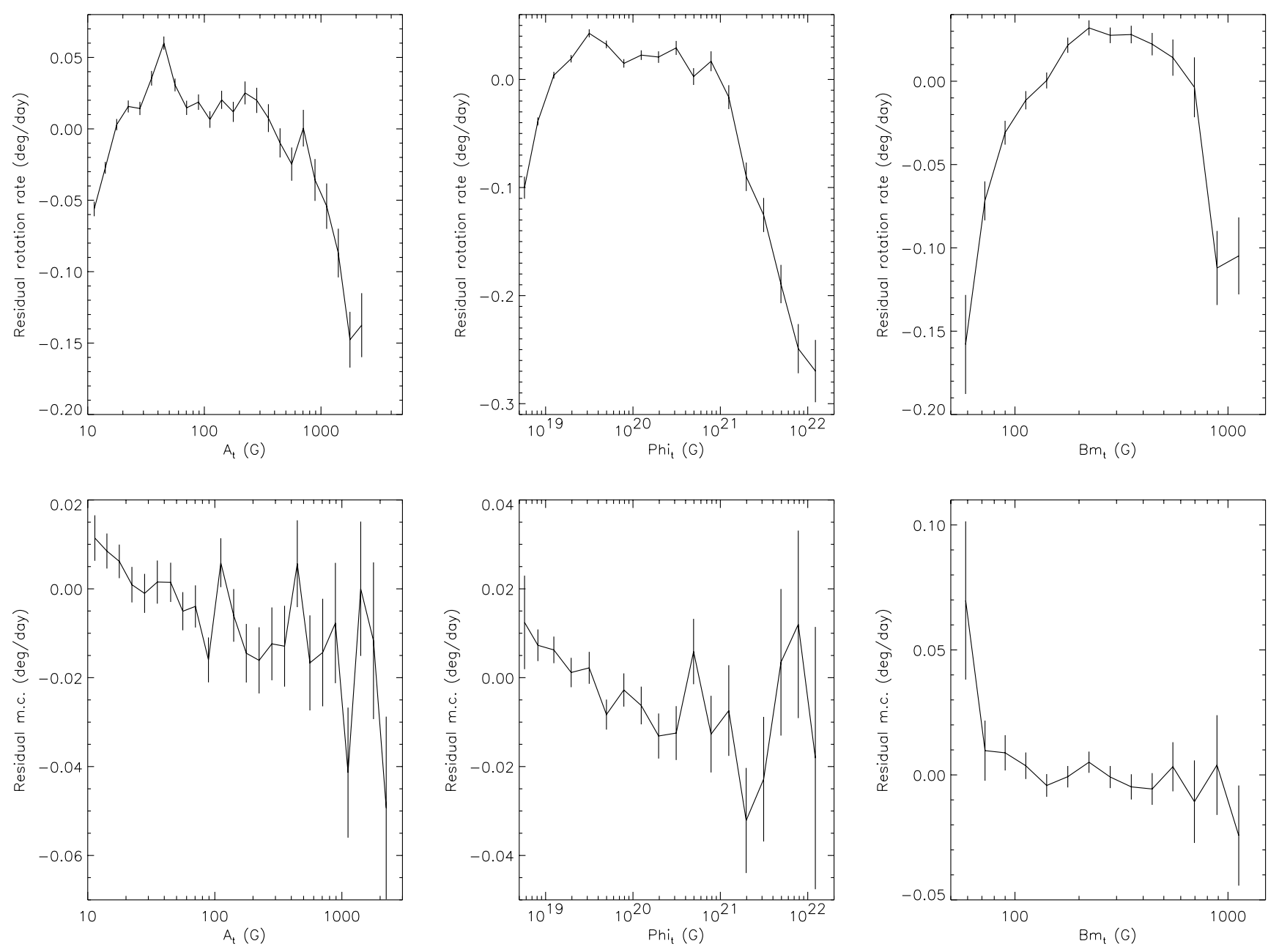

Fig. 7. Top: FT rotation residuals; Bottom: FT meridional circulation residuals. Left: versus $A_{\mathrm{t}}$. Middle: versus $\Phi_{\mathrm{t}}$. Right: versus $B m_{\mathrm{t}}$.

Table 2. FT residuals in $\operatorname{deg} / \mathrm{day}$ for $A$ in the range $2000-7000 \mathrm{Mm}^{2}$.

\begin{tabular}{ccc}
\hline \hline Dynamics & $B<900 \mathrm{G}$ & $B>900 \mathrm{G}$ \\
\hline Rotation rate & $-0.153 \pm 0.023$ & $-0.232 \pm 0.015$ \\
Meridional circulation & $0.018 \pm 0.021$ & $-0.002 \pm 0.014$ \\
\hline
\end{tabular}

are convergent flows inside a region, the CT will see them but not the FT. Also, CT is more sensitive to the strongest fields in the box, while the FT defines the region using only its contour, so CT will be more influenced by spot behavior for example.

The much smaller meridional circulation in quiet regions for FT compared to CT is probably real as well. In fact, if the FT method were biased toward lower values (the "reference" meridional circulation for the tracking is 0 ), it would also underestimate the equatorial meridional circulation of $\sim-0.05 \mathrm{deg} / \mathrm{day}$, which is not the case. Therefore it must represent a difference in meridional circulation for the individual magnetic structures and for the large scale pattern they constitute (such as supergranulation or organization on a larger scale).

Correspondence between the two methods can be established both ways. First, for a given correlation box, one can look at the average dynamics of the features inside that box. When a certain type of correlation box is selected, for example with $\left\langle\left|B_{\mathrm{c}}\right|\right\rangle$ in a certain range, the list of FT features corresponding to these boxes can be selected and the variation with size, for example, can be compared to the average one (not very different). Second, to each feature one can attribute the characteristic of the CT box in which this feature is present, including the dynamics and the magnetic field level. Such an analysis shows that FT and CT results are actually different: they are shifted despite the similarity between the curves in Figs. 7 and 8. This analysis confirms that the differences are real and not due to a simple selection effect for example; individual magnetic structures have different dynamics from their collective behavior organized in the magnetic network.

Finally, Table 3 shows the coefficients from a Legendre polynomial fit on the rotation rates versus latitude for various selections. Rotation is more differential for CT than for FT (both at low and high latitudes), and it is also more differential for weak field areas and small features. The asymmetry between the 2 hemispheres is very small and, in fact, almost insignificant for FT. For CT, a smaller rotation in the Northern hemisphere is found (mostly for low latitudes), which seems to be present mostly for weak field regions. 

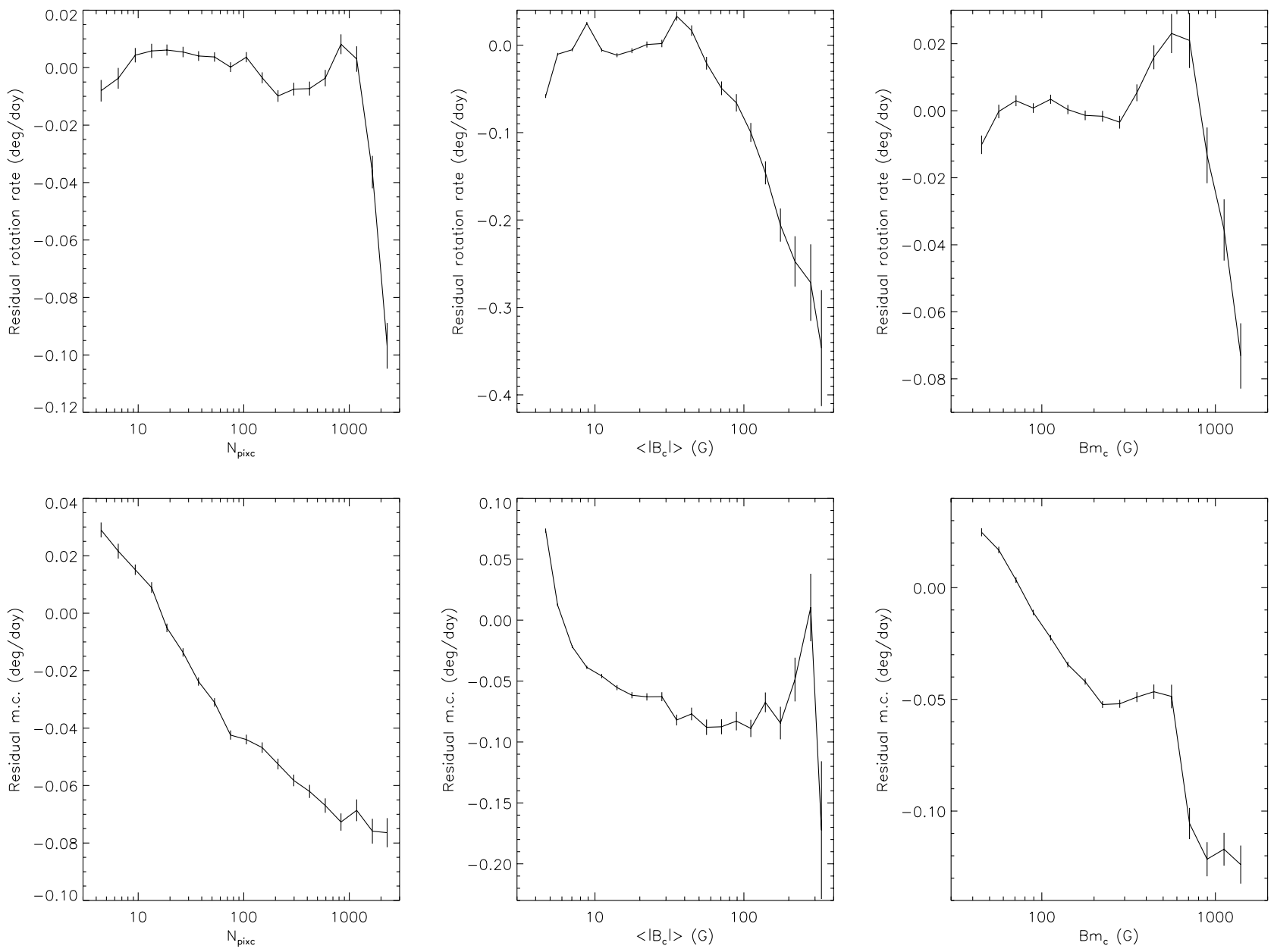

Fig. 8. Top: CT rotation residuals. Bottom: meridional circulation residuals. Left: versus $N$ pix ${ }_{\mathrm{c}}$. Middle: versus $\left\langle\left|B_{\mathrm{c}}\right|\right\rangle$. Right: versus $B m_{\mathrm{c}}$.

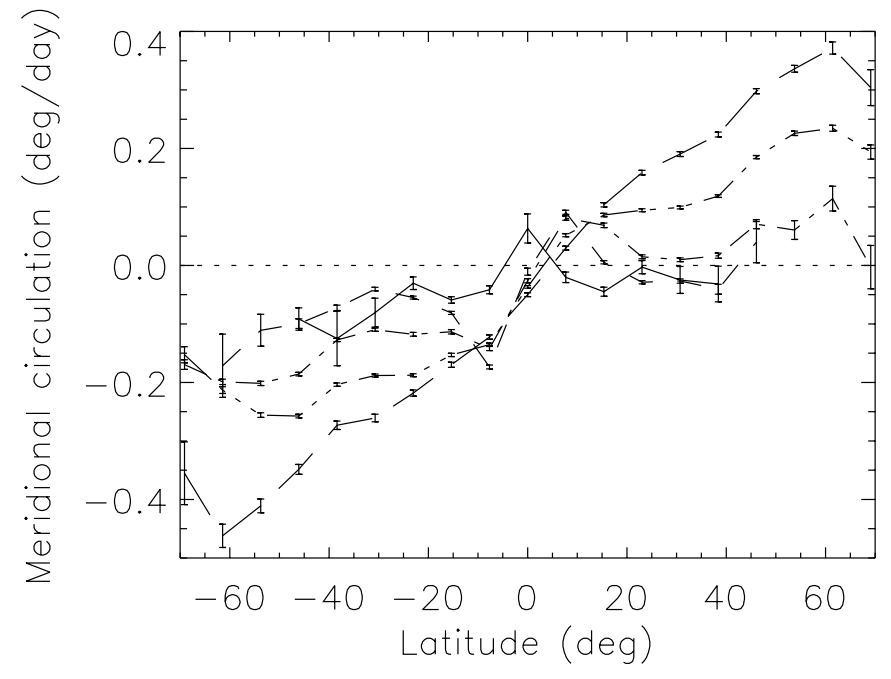

Fig. 9. CT meridional circulation residuals versus latitude for $\left\langle\left|B_{\mathrm{c}}\right|\right\rangle$ below $5 \mathrm{G}$ (long dashed), in the range 5-7 G (dot-dot-dot-dashed line), in the range 7-10 $\mathrm{G}$ (dot-dashed line), in the range 10-50 $\mathrm{G}$ (dashed line), and above $50 \mathrm{G}$ (solid line).

\subsection{Weak field dynamics and proximity to active regions}

It is interesting to check whether the small magnetic structures behave differently depending on their position with respect to active regions. For example, do small structures inside an active region have a meridional circulation such as that obtaind by the CT in these regions? To study this aspect, the dynamics of these structures is examined depending on their distance to large structures, as well as the dynamics for structures inside or outside a cluster of large structures.

\subsubsection{Dynamics and distance to large structures}

For each small structure, its distance to the closest large structure (with $A_{\mathrm{t}}>500 \mathrm{Mm}^{2}$ ) is computed. The dynamics of these small structures versus this distance can then be studied for various size ranges. The results are shown in Figs. 10 and 11.

Figure 10 shows the residual rotation rate and meridional circulation versus latitude for $A_{\mathrm{t}}<40 \mathrm{Mm}^{2}$ in two cases: structures very close to a large structure or far away from them. It shows that in active latitude areas, network features farther from active regions are rotating faster than similar features close to active regions (or inside it). Their meridional circulations are hardly different, however. For small structures close to active regions, no motion is observed like that of active regions with CT. The meridional circulation for structures far from active regions is more poleward above $20^{\circ}$ and less poleward close to the equator, compared to structure close to active 
Table 3. FT and CT coefficients from the Legendre polynomial fit performed on rotation rates versus latitude (such as in Fig. 4): $A_{1}$ and $A_{3}$ corresponds to the North-South asymmetry (respectively at low and high latitudes), and $A_{2}$ and $A_{4}$ corresponds to the differentiality of the rotation (at low and high latitudes respectively).

\begin{tabular}{ccc}
\hline \hline Selection & $A_{1}$ & $A_{3}$ \\
\hline FT & $0.013 \pm 0.012$ & $0.028 \pm 0.012$ \\
FT $\left(A<80 \mathrm{Mm}^{2}\right)$ & $-0.001 \pm 0.02$ & $0.01 \pm 0.01$ \\
FT $\left(A>500 \mathrm{Mm}^{2}\right)$ & $0.16 \pm 0.11$ & $0.14 \pm 0.09$ \\
CT & $-0.008 \pm 0.002$ & $-0.001 \pm 0.002$ \\
CT $\left(\left\langle\left|B_{\mathrm{c}}\right|\right\rangle<10 \mathrm{G}\right)$ & $-0.007 \pm 0.002$ & $0.002 \pm 0.003$ \\
$\mathrm{CT}\left(\left\langle\left|B_{\mathrm{c}}\right|\right\rangle>10 \mathrm{G}\right)$ & $0.007 \pm 0.012$ & $0.009 \pm 0.012$ \\
\hline Selection & $A_{2}$ & $A_{4}$ \\
\hline $\mathrm{FT}$ & $-2.21 \pm 0.03$ & $-0.34 \pm 0.02$ \\
FT $\left(A<80 \mathrm{Mm}{ }^{2}\right)$ & $-2.24 \pm 0.03$ & $-0.37 \pm 0.02$ \\
FT $\left(A>500 \mathrm{Mm}^{2}\right)$ & $-1.93 \pm 0.34$ & $-0.17 \pm 0.19$ \\
$\mathrm{CT}$ & $-2.568 \pm 0.003$ & $-0.552 \pm 0.003$ \\
$\mathrm{CT}\left(\left\langle\left|B_{\mathrm{c}}\right|\right\rangle<10 \mathrm{G}\right)$ & $-2.573 \pm 0.003$ & $-0.560 \pm 0.003$ \\
$\mathrm{CT}\left(\left\langle\left|B_{\mathrm{c}}\right|\right\rangle>10 \mathrm{G}\right)$ & $-2.289 \pm 0.025$ & $-0.360 \pm 0.016$ \\
\hline
\end{tabular}

regions. These structures far from active regions also tend to exhibit a convergence pattern as for active regions.

Figure 11 shows the variation of the residual rotation rate versus the distance for different sizes. The difference is mostly seen for features smaller than $40 \mathrm{Mm}^{2}$ and only up to a few degrees from the large structures, which confirms the previous result, with increasing rotation rates as the distance from the region increases. The influence of the active region remains closeby; note that $3^{\circ}$ corresponds to $36 \mathrm{Mm}$, i.e. like a large supergranule.

\subsubsection{Cluster analysis}

Large structures with $A_{\mathrm{t}}$ above $200 \mathrm{Mm}^{2}$ are grouped inside clusters by using a cluster analysis technique. The number of neighbors is computed within a given distance (taken as $19^{\circ}$, typical of the largest seperation in a bipolar region, from Howard 1992). If there are more than 2 neighbors, they are associated with the same cluster. This analysis over the whole data set, which concerns 81306 larger structures, leads to 6843 clusters, with an average of 8.5 large structures per cluster, and to $71 \%$ of the large structures inside this structure. This percentage increases slightly with size, up to $80 \%$ for the largest ones. Note that Pojoga \& Cudrik (2002) found that 40 to $50 \%$ of the active regions are inside clusters, when taking into account only those that would live more than 4 rotations. We find a slightly larger percentage here, but this is not surprising because we consider all structures. Using a distance criterion of $5^{\circ}$ (derived from Fig. 11), smaller structures can be associated to these clusters as well. We find 11.6 small structures inside these clusters. This percentage also increases from $\sim 10 \%\left(A_{\mathrm{t}} \sim 10 \mathrm{Mm}^{2}\right)$ to $24 \%\left(A_{\mathrm{t}} \sim 200 \mathrm{Mm}^{2}\right)$.
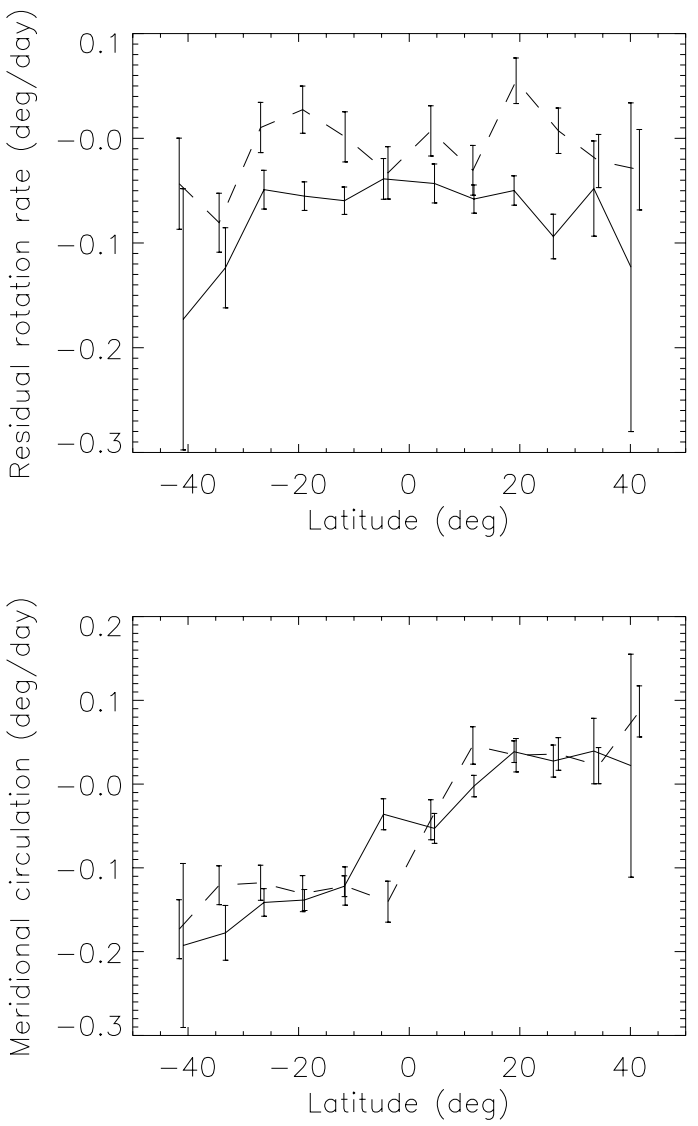

Fig. 10. FT residual rotation rates versus latitude for small distance to active region (below $5^{\circ}$, solid) and large distances (above $40^{\circ}$, dashed), for $A_{\mathrm{t}}$ below $40 \mathrm{Mm}^{2}$.

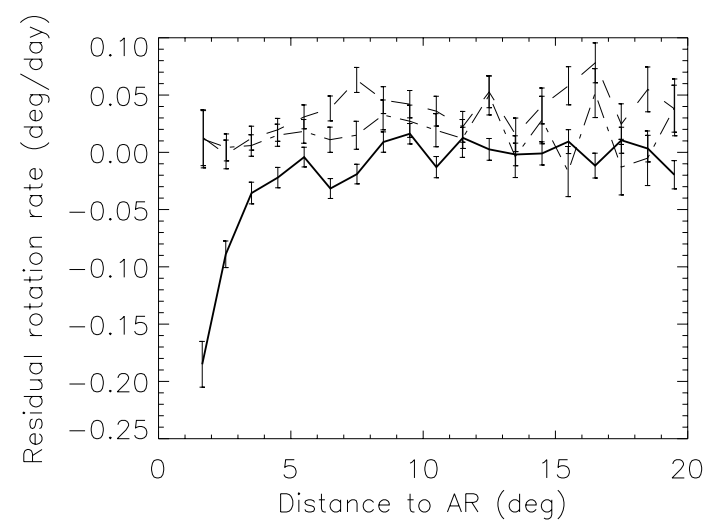

Fig. 11. FT residual rotation rate versus the distance to active regions, for $A_{\mathrm{t}}$ below $40 \mathrm{Mm}^{2}$ (solid thick line), in the range $40-80 \mathrm{Mm}^{2}$ (dashed line) and in the range $80-200 \mathrm{Mm}^{2}$ (dotted-dashed line).

Figure 12 confirms the faster rotation of small structures outside active regions defined here as clusters. This is seen only for small features (still significant when considering $A_{\mathrm{t}}<$ $200 \mathrm{Mm}^{2}$ ), not for larger ones. There may be a tendency to a less poleward meridional circulation of small structures outside clusters, but this is observed only in the southern hemisphere. 

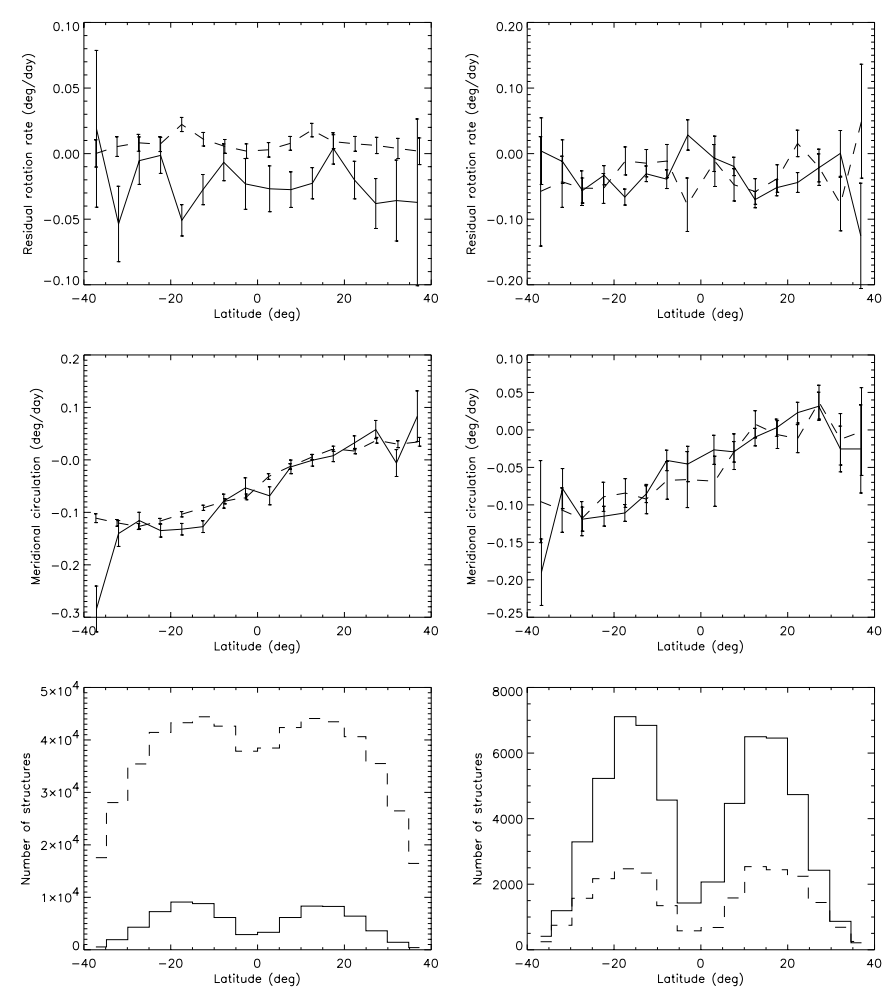

Fig. 12. FT residual rotation rates (top), FT meridional circulation (middle), and number of structures (bottom) versus latitude for structures inside a cluster (solid line) and outside (dashed line). Left: $A_{\mathrm{t}}<200 \mathrm{Mm}^{2} ;$ Right: $A_{\mathrm{t}}>200 \mathrm{Mm}^{2}$.

\section{Discussion and conclusion}

In this Paper I have studied the large scale dynamics of magnetic structures down to the network using a homogeneous data set covering most of a solar cycle. The two complentary methods (CT over a $93 \mathrm{Mm}$ scale and FT) should allow the anchoring depths of these features to be constrained.

The first new result is that CT provides a smaller rotation rate and larger meridional circulation than FT at all latitudes and almost all sizes. The rotation of structures observed with CT is likely to be related to supergranulation, but it is puzzling to see that the supergranular rotation rate is much larger (Duvall 1980; Snodgrass \& Ulrich 1990; Beck \& Schou 2000) and is in fact closer to the FT results than to the CT results (see Table 1). The meridional circulation in active areas using CT also exhibits a convergent pattern already observed previously (e.g. Meunier 1999; Haber et al. 2003; Zhao \& Kosovichev 2004). However, the small features associated with these active regions do not exhibit the converging pattern, but do exhibit a smaller rotation rate, as for large features. Furthermore, FT structures exhibit a small divergent pattern with respect to the latitude of the mean activity belt around active regions.

Given the increase in rotation rate observed by helioseismology as one goes inward (e.g. Schou et al. 1998, for example) down to $\sim 35-50 \mathrm{Mm}$, it is tempting to attribute a deeper anchoring to individual network features than to the pattern formed by these features. The decreasing meridional circulation usually observed by time-distance analysis
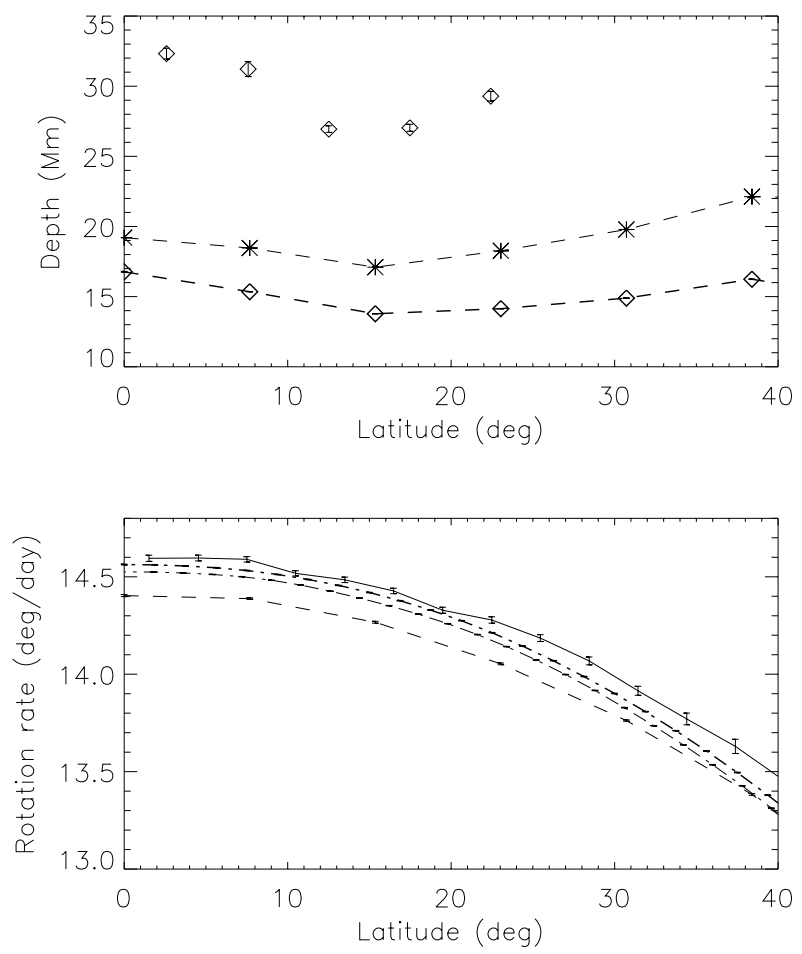

Fig. 13. Top: depth corresponding to FT rotation rate (diamonds with no line: GONG) and to the CT rotation rate (diamonds with thick dashed line: GONG; stars with thin dashed line: MDI) versus latitude. The depths correspond to the first intersection with the internal rotation rate from the surface. Internal rotation rates from helioseismology (MDI and GONG) are courtesy of R. Howe. Note that above $\sim 25^{\circ}$, no FT points for GONG are available since the FT rotation rate is larger than any internal rotation rate at that latitude. No FT point for MDI is available either, for the same reason. Bottom: rotation rate versus latitude for FT (solid line, $A$ in the range $40-50 \mathrm{Mm}^{2}$ ), for CT (dashed line, $\left\langle\left|B_{\mathrm{c}}\right|\right\rangle$ in the range $8-9 \mathrm{G}$ ), MDI (thin dotted-dashed line, maximum rate in the bulk of the convection zone), and GONG (thick dotted-dashed line, maximum rate in the bulk of the convection zone).

(Zhao \& Kosovichev 2004) or ring-diagram techniques (Haber et al. 2002; Komm et al. 2004) as one goes inward is consistent with this preliminary interpretation.

An attempt to attribute an anchoring depth to these structures is shown in Fig. 13. Internal rotation rates have been provided by R. Howe for MDI (OLA inversion) and GONG (RLS inversion). We concentrate on the results obtained below latitude $40^{\circ}$, because both data sets provide very different internal rotation rates at high latitudes (Schou et al. 2002). The FT and CT results are symmetrized with respect to the equator for this discussion. When using the MDI rotation rate, no anchoring depth can be attributed to the FT structures because the observed FT rate is too low; it is possible to attribute one for GONG, but only for the lowest latitudes (27-33 Mm). The CT rates beeing smaller, the attribution is easier to make, with depth in the range 13-17 Mm for MDI and 18-22 Mm for GONG. However, the impossibility of making the association for the FT rotation rates casts some doubt on the interpretation in term of anchoring. Figure 13 also shows the maximum rotation rate in the convection zone as a function of latitude. This rate is compared to the rate for FT structures that are rotating 
the fastest from (Fig. 7) and for the corresponding CT boxes from Fig. 8, and it also shows that most small FT structures rotate faster than any layer in the convection zone.

Another problem arises from the further constraint that can be derived from the observation of meridional circulation. Some features rotating with the same rate have a different meridonal circulation, for example with $\left\langle\left|B_{\mathrm{c}}\right|\right\rangle$ around $4 \mathrm{G}$ and $90 \mathrm{G}$, or features with $A \sim 10 \mathrm{Mm}^{2}$ and $\sim 1000 \mathrm{Mm}^{2}$. So this problem also concerns CT, despite the fact that Fig. 13 does not show any problem for that method. This is illustrated in Fig. 14, where a variation of the meridional circulation with depth can be deduced from the variation of meridional circulation with $\left\langle\left|B_{\mathrm{c}}\right|\right\rangle$, the variation of rotation rate with $\left\langle\left|B_{\mathrm{c}}\right|\right\rangle$, and the correspondence between our rotation rate and the internal rotation rate from helioseismology. This figure (bottom) shows clearly that the interpretation in terms of anchoring is not reasonable. If a structure is anchored at a certain depth so that its rotation rate is that of the layer in which it is anchored, we expect it to have the meridional circulation of that layer as well. Of course, a possibility would be that these two groups of structures are anchored in two different layers with a similar rotation rate (but a different meridional circulation). Helioseismology indeed shows that below $35-50 \mathrm{Mm}$ the internal rotation rate is decreasing again, so some of these features could be anchored above that layer, and the other below. However, it is quite unlikely that they could be anchored that deep. Indeed, recent results by Hiremath (2004) and Javaraiah \& Gokhale (1997) show that old spots always rotates in such a way that they are likely to be anchored above that turning point. Furthermore, it is likely that the rotation rates observed in this paper are related to supergranular flows, since the formation of network structures is associated with strong downflows on the edges of supergranules. However, it has been observed that the supergranulation pattern was probably present in a shallow layer only; for example, November (1994) found horizontal velocities in supergranules down to $2 \mathrm{Mm}$ only, with plumes associated with the strong downflows (therefore with the magnetic network) reaching $7 \mathrm{Mm}$. There is currently no evidence from helioseismology that a strong increase is present, followed by a decrease in the rotation rate close to the surface. Such a result has been observed by González Hernández \& Patron (2000), but they have not been observed in more recent works and are probably an artefact of the method (González Hernández, private communication).

The similarities in the curves of the rotation rate versus $A$ (FT) and versus $\langle|B|\rangle(\mathrm{CT})$ led us to think that they are correlated, however. Note that the increase for the weakest regions may be related to the recent results of Zhao et al. (2004), who have recently found that the surface rotation rate was higher for location with larger magnetic field up to $600 \mathrm{G}$. The amplitude is on the order of $70 \mathrm{~m} / \mathrm{s}$ over these $600 \mathrm{G}$. However, this does not necessarily represent the velocities of the magnetic features because of the filling factor smaller than 1 . Here we do observe this increase $(15 \mathrm{~m} / \mathrm{s})$ for features with size up to $40 \mathrm{Mm}^{2}$, corresponding to a flux of $3 \times 10^{19} \mathrm{Mx}$, which corresponds to an average magnetic field of $75 \mathrm{G}$, and maximum magnetic field of $200 \mathrm{G}$. For such an average magnetic field, Zhao et al. (2004) find a residual rotation rate around $17-18 \mathrm{~m} / \mathrm{s}$; therefore our
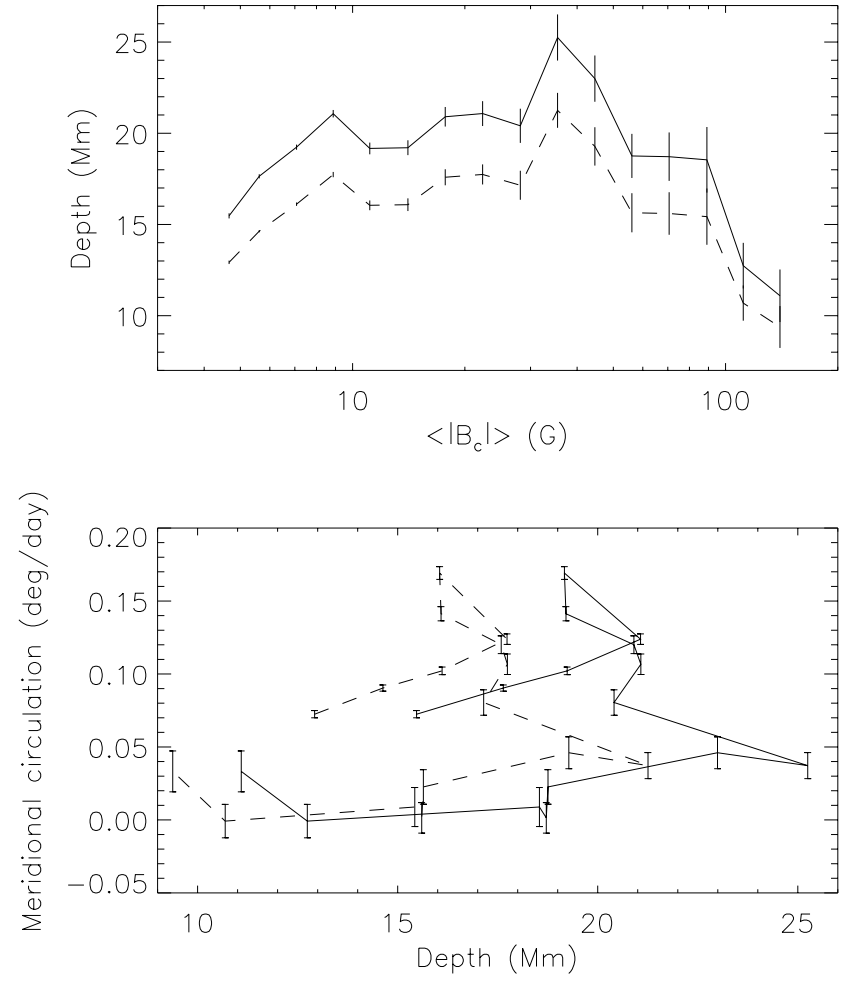

Fig. 14. Top: depth versus $\left\langle\left|B_{\mathrm{c}}\right|\right\rangle$ deduced from the CT rotation rate versus $\left\langle\left|B_{\mathrm{c}}\right|\right\rangle$, at latitude $\pm 7.68^{\circ}$ (first intersection with internal rotation rate from the surface), for MDI (solid line) and GONG (dashed line). Internal rotation rates from helioseismology (MDI and GONG) are courtesy of R. Howe. Bottom: meridional circulation versus depth deduced from the top curve and from the meridional circulation versus nedit $\left\langle\left|B_{\mathrm{c}}\right|\right\rangle$, at latitude $\pm 7.68^{\circ}$.

results are quite consistent with their rotation rate increase up to $200 \mathrm{G}$. Between 200 and $600 \mathrm{G}$, we would expect to see an increase above $40 \mathrm{Mm}^{2}$ (or $10 \mathrm{G}$ for CT), if this was due to a horizontal motion of the magnetic structures, which we do not see. Their errorbars above $200 \mathrm{G}$ are quite large, however.

Understanding these variations with magnetic level and their inconsistency with helioseismology should provide some clues about the origin of the magnetic network. An additional constraint is provided by the cluster analysis performed in this paper, which allows the dynamics of small features inside active regions to be compared with those of large structures in these regions, and with similar features far from active regions. I find that the smallest network features (below $40 \mathrm{Mm}^{2}$ ) tend to rotate slower than the others when they are located within $5^{\circ}$ of a large structure, but their rotation rate is not, however, as small as that of large structures. Their meridional circulation is independent of their location, as they do not exhibit this converging pattern when close to active regions. This shows that the smallest features belonging to active regions may be slightly influenced by the large structure behavior, as they would if they were resulting from their decay, but they do not behave in the same way. It is, however, too early to establish the anchoring depth of the FT and CT structures, either in absolute value or with respect to each other.

It is difficult to establish a complete comparison with all helioseismic results. First, the different analyses (time distance, 
ring diagrams) exhibit different results for various reasons: sensitivity to different layers for example, see also Hindman et al. (2004). However it is already possible to conclude that the anchoring of network structures at various depths cannot explain all characteristics, as their rotation rates and meridional circulation are not compatible with results from helioseismology. This does not mean that they cannot be anchored at a certain depth, but it is very likely that additional processes play a role in their dynamics and probably accelerate them. The excess of velocity would be in the range 30-60 m/s if they were anchored in the range 2-7 Mm (75-90 m/s for supergranules). Second, it has been shown (see e.g. Haber et al. 2002; Zhao \& Kosovichev 2004) that the meridional circulation seems highly variable; therefore it will be necessary to study its temporal variation in order to go further, an analysis to be presented in a separate paper (Meunier 2005b). The study of the dynamics versus polarities will also provide additional clues (Meunier 2005a).

Acknowledgements. I am very grateful to $\mathrm{R}$. Howe who has provided the internal rotation rates from helioseismic observations (GONG and MDI). I also thank the referee for suggesting the analysis of the flows with respect to the activity belt. SOHO is a mission of international cooperation between the European Space Agency (ESA) and NASA.

\section{References}

Antonucci, E., Azzarelli, L., Casalini, P., Cerri, S., \& Denoth, F. 1979, Sol. Phys., 63, 17

Brajša, R., Wöhl, H., Vršnak, B., et al. 2004, A\&A, 414, 707

Beck, J. G. 2000, Sol. Phys., 191, 47

Beck, J. G., \& Schou, J. 2000, Sol. Phys., 193, 333

D’Silva, \& Howard, R. 1994, Sol. Phys., 151, 213

Duvall, T. L., Jr. 1980, Sol. Phys., 66, 213

Giles, P. 1999, Ph.D. Thesis, Stanford University
González Hernández, I., \& Patron, J. 2000, Sol. Phys., 191, 37

Haber, D. A., Hindman, B. W., \& Toomre, J. 2003, in Proc. SOHO 12/GONG+ 2002, Local and Global Helioseismology: The Present and Future, ed. H. Sawaya-Lacoste, 103

Haber, D. A., Hindman, B. W., Toomre, J., et al. 2002, ApJ, 570, 855

Hindman, W. B., Gizon, L., Duvall, T. L., Jr., Haber, D. A., \& Toomre, J. 2004, ApJ, 613, 1253

Hiremath, K. M. 2002, A\&A, 386, 674

Howard, R. 1990, Sol. Phys., 126, 299

Howard, R. 1991, Sol. Phys., 135, 327

Howard, R. 1992, Sol. Phys., 142, 233

Howard, R. 1996, ARA\&A, 34, 75

Javaraiah, J., \& Gokhale, M. H. 1997, A\&A, 327, 795

Komm, R. W., Howard, R. F., \& Harvey, J. W. 1993a, Sol. Phys., 145, 1

Komm, R. W., Howard, R. F., \& Harvey, J. W. 1993b, Sol. Phys., 147, 203

Komm, R. W., Corbard, T., Durney, B. R., et al. 2004, ApJ, 605, 554

Meunier, N. 1999, ApJ, 527, 967

Meunier, N. 2003, A\&A, 405, 1107

Meunier, N. 2005a, A\&A, in press

Meunier, N. 2005b, submitted

Meunier, N., Nesme-Ribes, E., \& Grosso, N. 1997a, A\&A, 319, 673

Meunier, N., Nesme-Ribes, E., \& Collin, B. 1997b, A\&A, 319, 683

November, L. J. 1994, Sol. Phys., 154, 1

Pojoda, S., \& Cudrik, B. 2002, Sol. Phys., 208, 17

Sivaraman, K. R., \& Gokhale, M. H. 2004, Sol. Phys., 220, 209

Snodgrass, H. B., \& Ulrich, R. K. 1990, ApJ, 351, 309

Scherrer, P. H., Bogart, R. S., Bush, R. I., et al. 1995, Sol. Phys., 162, 129

Schou, J., Howe, R., Basu, S., et al. 2002, ApJ, 567, 1234

Strous, L. H. 2000, Sol. Phys., 195, 219

Wöhl, H. 2002, Astr. Notes, 323, 329

Wöhl, H., \& Brajša, R. 2001, Sol. Phys., 198, 57

Zhao, J., \& Kosovichev, A. G. 2004, ApJ, 603, 776

Zhao, J., Kosovichev, A. G., \& Duvall, T. L., Jr. 2004, ApJ, 607, L135 